Portland State University

PDXScholar

Electrical and Computer Engineering Faculty

Publications and Presentations

$2-1-2002$

\title{
Forces on Permeable Conductors in Magnetic Fields
}

Lee W. Casperson

Portland State University

Follow this and additional works at: https://pdxscholar.library.pdx.edu/ece_fac

Part of the Electrical and Computer Engineering Commons

Let us know how access to this document benefits you.

Citation Details

Casperson, L. W. (2002). Forces on permeable conductors in magnetic fields. American Journal of Physics, 70(2), 163-168.

This Article is brought to you for free and open access. It has been accepted for inclusion in Electrical and Computer Engineering Faculty Publications and Presentations by an authorized administrator of PDXScholar. Please contact us if we can make this document more accessible: pdxscholar@pdx.edu. 


\title{
Forces on permeable conductors in magnetic fields
}

\author{
Lee W. Casperson ${ }^{\text {a) }}$ \\ Departments of Electrical and Computer Engineering and of Physics, Portland State University, \\ P.O. Box 751, Portland, Oregon 97207-0751
}

(Received 2 April 2001; accepted 9 October 2001)

\begin{abstract}
It is often implied that the force density formula $d \mathbf{F} / d \mathbf{V}=\mathbf{J} \times \mathbf{B}$ is all that is required to calculate the force that would be experienced by any stationary current-carrying medium in a region of space containing a magnetic field. However, representations of this formula are not all compatible, and the methods of applying such formulas when the conductor or surrounding medium have permeabilities different from vacuum are not widely known. The simplest case that one might consider is that of a current-carrying wire in an otherwise uniform field. It appears that the experimental measurements corresponding to such a situation have not been carried out for permeable media, and these results are reported here. The permeability and current can cause substantial changes in the field distribution from its background form, but the total force per unit length on the wire remains compatible with the formula $\mathbf{I} \times \mathbf{B}_{0}$, with $\mathbf{I}$ being the conduction current and $\mathbf{B}_{0}$ being the flux density that was present before the permeable current-carrying wire was introduced. (C) 2002
\end{abstract} American Association of Physics Teachers.

[DOI: $10.1119 / 1.1424265$ ]

\section{INTRODUCTION}

There is an abundance of modern literature on electromagnetics, yet there remain ambiguities concerning the form and use of the force equations. One of the simplest experiments that one might imagine for testing a force formula for conducting media involves measurements on a current-carrying wire in an otherwise uniform magnetic field. Such measurements would be helpful to clarify discrepancies between theoretical models for the case that either the wire or the surrounding medium has a permeability different from the permeability of free space. In this study we have considered several models and measured the force on a permeable current-carrying wire in a magnetic field. ${ }^{1}$ This fundamentalsounding subject seems not to have been explored experimentally, and the results provide insight into the validity and use of various force formulas. The experimental setup described here should also be adaptable to other measurements involving weak electric or magnetic forces in the presence of other substantial fields.

A brief sketch of some of the earliest studies of forces on moving point charges and currents is given in Sec. II. It is seen that these results are ambiguous, and previous experimental tests relating to volume currents have involved media with near-vacuum permeabilities. The electromagnetic fields in the vicinity of a current-carrying wire are reviewed in Sec. III, and the implications of these fields for the forces on the conductors are also considered. Experimental force measurements on wires of different permeabilities are reported in Sec. IV. The measurements agree with the most familiar force laws only if one ignores contributions to the magnetic field arising from the current and permeability of the wire. Certain more advanced interpretations are, however, in agreement with the experimental results.

\section{BACKGROUND}

Formulas for the forces on current-carrying conductors in magnetic fields were discussed by Maxwell as part of his comprehensive studies of electricity and magnetism, published in 1873. Maxwell's results included an expression for the force on a circuit element carrying a current $\mathbf{I}$ in a region of magnetic flux density $\mathbf{B}$. In modern notation his formula for the force per unit length acting on the line current can be written in the form ${ }^{2}$

$$
\frac{d \mathbf{F}}{d l}=\mathbf{I} \times \mathbf{B} .
$$

As an alternative to charged fluid interpretations of current flow, some researchers proposed that electric currents might represent the flow of discrete electrical charges. Based on studies of currents in evacuated tubes, C. F. Varley suggested in 1871 that cathode rays consisted of streams of negatively charged particles. ${ }^{3}$ This hypothesis was supported in further studies by A. Schuster in $1884 .{ }^{4}$ He showed that the force on a charged particle moving perpendicular to the magnetic field would cause the particle to be deflected into a circular trajectory, and in modern notation his results would correspond to the formula

$$
\mathbf{F}=q \mathbf{v} \times \mathbf{B},
$$

where $q$ is the charge on the particle. Theoretical studies of the magnetic force on moving electric charges were also carried out by J. J. Thomson in $1881 .^{5} \mathrm{O}$. Heaviside in his 1889 investigations of this subject referred to the force on a current element or point charge as Maxwell's electromagnetic force. ${ }^{6}$ In 1890 Schuster combined the magnetic force formula with the centripetal force formula and detailed cathode ray deflection experiments to obtain an estimate of the charge-to-mass ratio of a cathode ray particle (electron). ${ }^{7}$

Without referencing earlier work, Lorentz in 1895 also argued that charge and current effects result from the existence and motion of small negatively charged particles. ${ }^{8} \mathrm{He}$ considered that all fields exist in empty space (ether), and within this space there is only one electric vector (he chose electric displacement $\mathbf{D}$ rather than electric field $\mathbf{E}$ ) and one magnetic vector (he chose $\mathbf{H}$ rather than B). Like Schuster, he recognized that an electric charge in motion may be acted on by a force proportional to its velocity and directed at right angles to it. In modern notation Lorentz's equation for the force acting on a point charge would be written in the form 


$$
\mathbf{F}=q \mathbf{v} \times \mu_{0} \mathbf{H},
$$

where $\mu_{0}$ is the permeability of free space. In vacuum the magnetic field vectors are connected by the simple relationship $\mathbf{B}=\mu_{0} \mathbf{H}$, so the force laws given in Eqs. (2) and (3) are equivalent for this special case. Lorentz also understood that a continuous stream of electrons would correspond to a current, and the force per unit length acting on a current element would be governed by ${ }^{8}$

$$
\frac{d \mathbf{F}}{d l}=\mathbf{I} \times \mu_{0} \mathbf{H} .
$$

In vacuum this result is equivalent to Eq. (1).

The most striking feature of the force equations for point charges and line currents is their inconsistency for media having other than vacuum permeability. In some ferromagnetic materials, for example, the discrepancy between $\mu_{0} \mathbf{H}$ and $\mathbf{B}$ can be several orders of magnitude. It was considered appropriate to resolve this discrepancy by means of experiment. Starting in 1931, several experiments were undertaken involving the transmission of electrons and other particles through magnetic media. ${ }^{9-17}$ These experiments have been interpreted to demonstrate that for particles not moving too slowly, the $\mathbf{B}$ form of the force law given in Eq. (2) is correct.

The force formulas have generalizations to other current density distributions. The corresponding results for a volume current density $\mathbf{J}$ would seem to be either

$$
\frac{d \mathbf{F}}{d V}=\mathbf{J} \times \mathbf{B} \quad(B \text { form })
$$

or

$$
\frac{d \mathbf{F}}{d V}=\mathbf{J} \times \mu_{0} \mathbf{H} \quad(H \text { form }),
$$

where $d \mathbf{F} / d V$ now represents the force per unit volume. These formulas appear widely in the literature. Although Eq. (2) may often be usable for the propagation of isolated particles through magnetic media, it doesn't follow that the corresponding formula for macroscopic current densities should also be valid in the form given in Eq. (5). As discussed below, other procedures have also been proposed for determining the force on current-carrying media in magnetic fields. Thus, an experimental test of these procedures would seem to be appropriate, and such a test is one of the main purposes of this study. By analogy, experiments were also necessary to determine whether the couple on a bar magnet in a magnetic field depends on $\mathbf{H}$ or on $\mathbf{B} .^{18}$

\section{FIELDS AND FORCES}

The experimental configuration of interest here is shown in schematic cross section in Fig. 1. A wire carrying a uniform current density directed into the plane of the figure is centered between two parallel and identically oriented permanent magnets. If the spacing between the magnet faces is small compared to their transverse dimensions, the field between the faces would, in the absence of the wire, be approximately uniform, and fringing and external field effects can be neglected. Furthermore, if the wire diameter is small compared to the magnet spacing, this configuration corresponds approximately to a current-carrying wire in an otherwise uniform magnetic field. When the current flows through

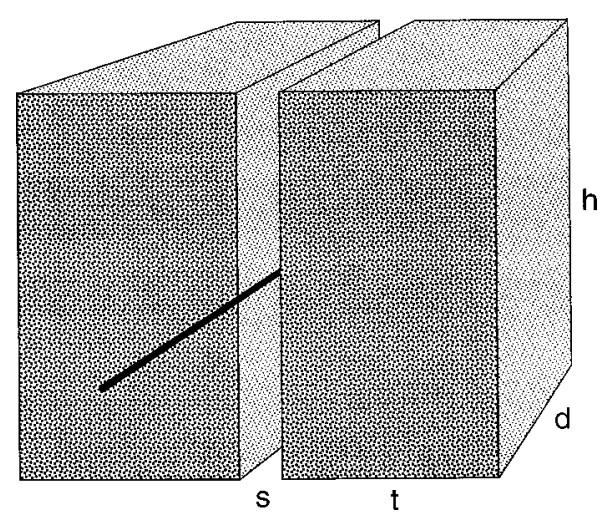

Fig. 1. Representation of a cylindrical wire carrying a current across the nearly parallel field lines between two permanent magnets. The letters $s, t, d$, and $h$ denote the spacing, thickness, depth, and height of the magnets, respectively.

the wire, it will experience a force in the up or down direction, while a force in the opposite direction is experienced by the magnets. This arrangement provides a relatively simple means for investigating the force law for a current-carrying permeable medium in a magnetic field, and it corresponds directly to the experiment described in Sec. IV.

To carry out a field calculation for the configuration shown in Fig. 1, it is first necessary to establish an appropriate coordinate system. The rectangular and cylindrical coordinates to be used here are indicated in the sketch given in Fig. 2. The $z$ axis corresponds to the axis of the wire and is directed out of the plane of Fig. 2. The inside of the conductor is region 1, and the outside is region 2. Most of the analysis would typically be carried out in a cylindrical coordinate system, but rectangular coordinates are helpful in interpreting the uniform fields that sometimes occur.

The field at large distances is assumed to be constant, and we express this field in the form

$$
\mathbf{H}_{\infty}=H_{0} \mathbf{i}_{x} .
$$

In cylindrical coordinates $\mathbf{H}_{\infty}$ is

$$
\mathbf{H}_{\infty}=H_{0}\left(\mathbf{i}_{r} \cos \phi-\mathbf{i}_{\phi} \sin \phi\right) .
$$

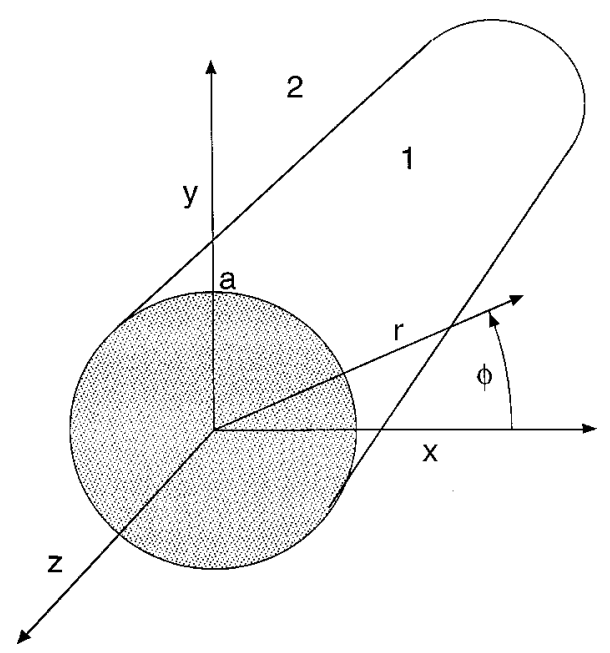

Fig. 2. Rectangular and cylindrical coordinate systems for studying the magnetic fields and forces in the vicinity of a current carrying wire in an external magnetic field. Region 1 is inside of the wire, and region 2 is outside. 
From Maxwell's equations, the magnetic fields in the two regions due only to the distant external sources can be written as

$$
\begin{aligned}
\mathbf{H}_{1 \text { ext }}= & \mathbf{i}_{r} \frac{2 \mu_{2}}{\mu_{1}+\mu_{2}} H_{0} \cos \phi-\mathbf{i}_{\phi} \frac{2 \mu_{2}}{\mu_{1}+\mu_{2}} H_{0} \sin \phi \\
= & \mathbf{i}_{x} \frac{2 \mu_{2}}{\mu_{1}+\mu_{2}} H_{0}, \\
\mathbf{H}_{2 \text { ext }}= & \mathbf{i}_{r}\left(1+\frac{\mu_{1}-\mu_{2}}{\mu_{1}+\mu_{2}} \frac{a^{2}}{r^{2}}\right) H_{0} \cos \phi \\
& -\mathbf{i}_{\phi}\left(1-\frac{\mu_{1}-\mu_{2}}{\mu_{1}+\mu_{2}} \frac{a^{2}}{r^{2}}\right) H_{0} \sin \phi .
\end{aligned}
$$

The second form of Eq. (9) shows that for this case the internal field is uniform and in the same direction as the externally applied field. It is also clear from these results that if the internal and external permeabilities are equal $\left(\mu_{1}\right.$ $=\mu_{2}$ ), the field is uniform everywhere. If the external permeability is small compared to the permeability of the wire $\left(\mu_{2} \ll \mu_{1}\right)$, the magnetic field inside the wire is small compared to the magnetic field far away, while the flux density inside is twice as large as the flux density at large distances. On the other hand, if the permeability of the wire is small compared to the permeability outside $\left(\mu_{2} \gg \mu_{1}\right)$, the field in the wire is twice the field far away while the flux density inside is small compared to the flux density at large distances.

The magnetic fields due to the current in the conducting region may also readily be found. If the current density is uniform over the cross section of the conductor, the currentcaused field inside is given by

$$
\mathbf{H}_{1 \mathrm{cur}}=\mathbf{i}_{\phi} \frac{r J}{2}, \quad r<a,
$$

while the corresponding field outside is

$$
\mathbf{H}_{2 \mathrm{cur}}=\mathbf{i}_{\phi} \frac{a^{2} J}{2 r}, \quad r>a .
$$

If we combine Eqs. (11) and (12) with Eqs. (9) and (10) for external field sources, we find that the total field everywhere is given by

$$
\begin{aligned}
\mathbf{H}_{1}= & \mathbf{i}_{r} \frac{2 \mu_{2}}{\mu_{1}+\mu_{2}} H_{0} \cos \phi+\mathbf{i}_{\phi}\left(\frac{r J}{2}-\frac{2 \mu_{2}}{\mu_{1}+\mu_{2}} H_{0} \sin \phi\right) \\
= & \mathbf{i}_{x} \frac{2 \mu_{2}}{\mu_{1}+\mu_{2}} H_{0}+\mathbf{i}_{0} \frac{r J}{2}, \\
\mathbf{H}_{2}= & \mathbf{i}_{r}\left(1+\frac{\mu_{1}-\mu_{2}}{\mu_{1}+\mu_{2}} \frac{a^{2}}{r^{2}}\right) H_{0} \cos \phi \\
& +\mathbf{i}_{\phi}\left[\frac{a^{2} J}{2 r}-\left(1-\frac{\mu_{1}-\mu_{2}}{\mu_{1}+\mu_{2}} \frac{a^{2}}{r^{2}}\right) H_{0} \sin \phi\right] .
\end{aligned}
$$

Having the magnetic fields in the vicinity of a currentcarrying wire, it should be possible to calculate the magnetic forces acting on that wire. These calculations can be done in various ways, and we consider first the possibilities implied in Sec. II. In the simplest situations the wire and the surrounding medium both have permeabilities close to that of free space, $\mu_{0}$. Copper, for example, has a permeability of about $\mu_{\text {copper }}=0.999991 \mu_{0}$, while the permeability of air is about $\mu_{\text {air }}=1.0000004 \mu_{0} \cdot{ }^{19}$ This case corresponds to the experiments of Ampere and his successors. The force laws given in Eqs. (5) and (6) are essentially identical for these media. With Eq. (5) or (6) for the force density and Eq. (13) for the magnetic field (with $\mu_{2}=\mu_{1}=\mu_{0}$ ), the total force per unit length acting on the cylindrical wire would seem to be

$$
\frac{d \mathbf{F}}{d l}=\int_{0}^{2 \pi} \int_{0}^{a} \frac{d \mathbf{F}}{d V} r d r d \phi=\mathbf{i}_{y} \pi a^{2} J \mu_{0} H_{0}=\mathbf{i}_{y} I \mu_{0} H_{0},
$$

where $I=\pi a^{2} J$ is the total current in the wire.

Real wires would have permeabilities that are at least slightly different from $\mu_{0}$, and most books on electromagnetics state without qualification that the force density on a current-carrying medium in a magnetic field is given by Eq. (5). If one uses Eq. (5) for the force density with Eq. (13) for the field, then Eq. (15) for the total force per unit length would be replaced in this analysis by the result

$$
\frac{d \mathbf{F}}{d l}=\mathbf{i}_{y} I \frac{2 \mu_{1} \mu_{2}}{\mu_{1}+\mu_{2}} H_{0} .
$$

On the other hand, if we use Eq. (6), the force per unit length would be

$$
\frac{d \mathbf{F}}{d l}=\mathbf{i}_{y} I \frac{2 \mu_{0} \mu_{2}}{\mu_{1}+\mu_{2}} H_{0} .
$$

Clearly, these formulas differ substantially from each other and from Eq. (15) for a macroscopic permeable conductor.

Other more complicated but less known formalisms for determining the force on permeable current-carrying media have also been developed, but their relevance and accuracy have also not been established in the laboratory. In energybased models the forces are derived by means of stress tensors, and one finds from such treatments that the force densities given above need to be supplemented with terms resulting from spatial variations of the permeability. For the particular case of a wire in a magnetic field, the permeability may change abruptly at the wire surface resulting in substantial surface forces. Inclusion of these forces suggests that the force per unit length, $\mathbf{I} \times \mathbf{B}_{0}$, should still be correct even if the wire has a nonvacuum permeability. ${ }^{1,20,21}$

The magnetic forces can also be calculated if the magnetic medium is represented as a distribution of magnetic poles, dipoles, or magnetization currents. ${ }^{22-25}$ In the magnetization current picture, for example, one adopts the point of view that all of the magnetic effects of the medium result from the real conduction currents together with bound currents representing the microscopic dipole moment density. As the totality of these currents is used to calculate the magnetic field, one may in a self-consistent way include this same total current in the force density equation. Using this approach, one finds that the modifications to the force density resulting from changes in the field and current distributions all cancel. Thus, the force per unit volume of Eq. (5) also leads to the force per unit length in the form $\mathbf{I} \times \mathbf{B}_{0}$, provided that $\mathbf{J}$ in Eq. (5) is now the sum of the conduction current and the magnetization current. Equivalent results may also be obtained if the magnetization is represented as a distribution of magnetic poles or dipoles. ${ }^{24,25}$ Given these varied results and 


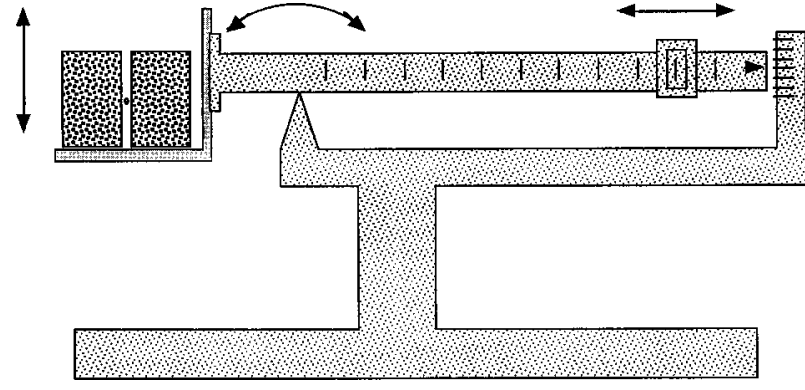

Fig. 3. Schematic representation of a modified balance for measuring the force on the magnets at the left due to the current carrying wire between them. The arrows indicate the motions associated with the use of this balance. The vertical arrows indicate the up and down sensitivity adjustment of the magnets with respect to the balance beam, and the curved arrows suggest the possible angular motion of the beam on the knife-edge fulcrum as balance is being achieved with right and left adjustments of the weights on the right-hand arm of the balance. The state of the balance is read from the independently supported scale on the right.

interpretations, experimental measurements would seem to be a reasonable step toward identifying or confirming a correct procedure.

\section{EXPERIMENT}

The essence of our experimental setup is shown in Fig. 1. A cylindrical current-carrying wire passes between two parallel neodymium-iron magnets. As discussed above, the resulting interaction will cause the wire to be pulled up and the magnets down or vice versa, depending on the relative directions of the current and the magnetic field.

Because of additional complicated forces if the currentcarrying wires are flexed or under stress in the measurement process, we have chosen to have the wires anchored while the force on the magnets is measured. Also, to minimize uncertainties that might occur with other types of forcemeasuring apparatus operating near intense magnetic fields, our setup is based on a modified version of a knife-edge balance with which all parameters can be monitored and adjusted. This system has the desirable features of allowing null measurements with only a single degree of freedom, so that any undesirable forces can readily be balanced out. Specifically, we have started from a Model 311 Ohaus Cent-OGram four-beam balance having a sensitivity of $0.01 \mathrm{~g}$. Our principal modifications include adding a larger base supporting the wire positioning system, and replacing the sample pan with an adjustable support for the two magnets. The wire positioning system includes Newport translation stages for precise placement and orientation of the tensioned wire. The magnet support on the balance allows vertical adjustment of the magnet pair with respect to the end of the beam. The magnet adjustment arrangement and the horizontal motion of the sliding weights are represented schematically in Fig. 3.

The vertical magnet adjustment provides two important functions with respect to the balance sensitivity. Varying the height of the magnets varies the height of the center of mass of the overall balance assembly, and higher mass tends to bring greater sensitivity. However, if the center of mass is higher than the knife-edge support, the balance becomes unstable. This adjustment also allows compensation because the magnet faces are flat while they rotate in a circular motion about the knife-edge balance axis. Thus, rotation in either direction moves the wire closer to the right-hand magnet and farther from the left-hand magnet in Fig. 3. If the wire has a permeability that is large compared to vacuum ( $\mu$ $\left.\gg \mu_{0}\right)$, the field structure of the magnets will, with rotation, cause a slight excess attraction toward the nearer right-hand pole face. This attraction with increasing angular displacement can also tend to destabilize the balance. This destabilization is compensated by lowering the magnet assembly on the balance arm, and in our experiments the magnet position was chosen in a way that retains the overall 0.01 -g sensitivity of the balance readings. Note that in our experimental implementation of the setup shown in Fig. 3, the distance between the wire and the knife-edge balance point is different from the original distance between the balance pan support and the knife edge. This difference would lead to a systematic error in the absolute mass calibration of the balance. However, this error wouldn't affect our principal conclusions, and it is corrected with an obvious rescaling of the mass readings.

We have performed several measurements with this system using wires of various sizes and compositions and various currents. In principle though, only two measurements are needed to distinguish between the force formulas for a permeable current-carrying wire in a magnetic field. The first measurement could use, for example, a copper wire ( $\mu$ $\approx \mu_{0}$ ) and would serve as a reference to experimentally calibrate the magnets and balance. The second measurement could use a steel wire $\left(\mu \gg \mu_{0}\right)$ to establish the correct force formula for permeable media. To better understand our experiments, it may be useful to indicate the actual numerical values for the parameters involved.

The magnets have a thickness in the direction parallel to the balance beam of $15.0 \mathrm{~mm}$, a depth perpendicular to the beam of $20.0 \mathrm{~mm}$, and a height of about $27.5 \mathrm{~mm}$. The copper wire used in the calibration experiments described below has a diameter of about $0.406 \mathrm{~mm}$. This wire is positioned at a distance from the knife edge that is chosen to be 2.00 times the original distance of the balance pan support. Thus, any mass measurements read from the beams' sliding weights should be scaled down by a factor of one-half. A second set of measurements was performed with a steel wire having a diameter of about $0.257 \mathrm{~mm}$. Because of its high permeability, the steel wire is drawn very strongly into regions of high magnetic field. Consequently, adequate tensioning and careful initial positioning adjustments are required to zero out forces due to any slight fringing effects of the fields.

Results from these two sets of measurements are shown in Fig. 4. The mass values determined from the balance readings (before scale correction) are plotted as a function of the current through the wire for the copper and steel wires. The most obvious implications of this figure are that the force varies linearly with the current and that there is little or no difference between the results obtained for copper and for steel. Thus, the force is always in agreement with the formula $\mathbf{I} \times \mathbf{B}_{0}$, where $\mathbf{B}_{0}$ is the flux density that was present before the permeable wire was introduced. It may be noted that our ability to set the current at multiples of $100 \mathrm{ma}$ is accurate to about $1 \%$, while the reading accuracy of the balance is about $0.01 \mathrm{~g}$. In view of these uncertainties, the small discrepancies in Fig. 4 between the results for copper and steel are not significant.

\section{CONCLUSION}

In this study we have considered the problem of the forces on a cylindrical conductor with nonvacuum permeability in 


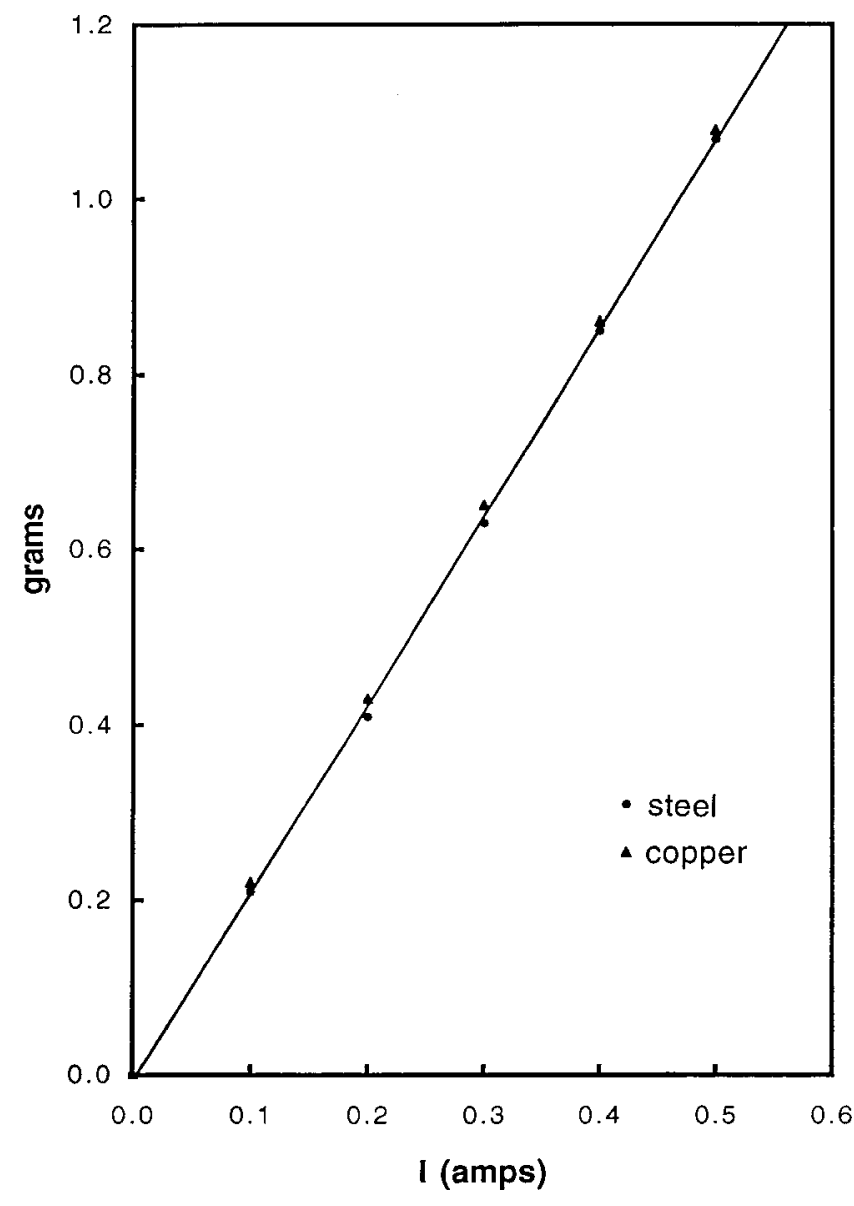

Fig. 4. Mass readings from the modified balance as a function of current through the wire for copper and steel wires.

an externally applied magnetic field. Based on most modern electromagnetics texts, it would appear that these forces may be fully described starting from the formula $d \mathbf{F} / d V=\mathbf{J} \times \mathbf{B}$ or possibly $d \mathbf{F} / d V=\mathbf{J} \times \mu_{0} \mathbf{H}$. The background of these equations has been reviewed, and their implications for a wire in a magnetic field have been treated in detail. Because these and other models do not appear to be fully compatible, we also have carried out experimental investigations of these formulas for permeable conductors.

Our experiments reveal clearly that the above formulas with their generally implied interpretation are both inadequate except for free space permeabilities, and it is unfortunate that they are so widely and uncritically presented. That the force should vary linearly with current is in agreement with all of the models described in Sec. III. On the other hand, Eq. (5) as written only yields the correct result if the flux density in the formula is assumed to have the value that was present before the permeable current-carrying wire was introduced, and while not obvious for macroscopic media, this requirement is seen to be analogous to the way in which the point charge force formulas are employed.

Other theoretical models have also been developed for the study of forces on current-carrying media, and some of these models yield results that are in complete agreement with our experiments. Thus, in some treatments the current density in the force formulas is understood to include magnetization currents in addition to conduction currents, while in others the force formulas themselves are modified to include the effects of permeability gradients which then lead to surface forces. Tensor methods have also proven to be effective for calculating the forces on conductors. The experiments reported here provide a means for excluding incorrect force formulas or interpretations.

As a final note, it may be observed that the bound currents in the magnetization current model for the magnetic properties of a medium do not necessarily correspond to actual physical electric currents. At the atomic level, magnetic properties result both from charge motion and from the magnetic dipole moments associated with the intrinsic spins of the constituent fundamental particles. Thus, ferromagnetic polarization is due largely to electron-spin magnetic moments. ${ }^{26}$ At this more fundamental level, semiclassical electric current and dipole moment densities for the electrons can be derived from the wave function solutions of Dirac's equation, ${ }^{27}$ and these densities can then be incorporated into Maxwell's equations for the calculation of microscopic or macroscopic fields. At a still smaller level, proton magnetization is now understood to be due in part to the spins of the constituent quarks and gluons as well as to motion of the electrically charged quarks. ${ }^{28}$

${ }^{a}$ Electronic mail: 1caspers@ee.pdx.edu

${ }^{1}$ L. W. Casperson, "Forces on permeable conductors in magnetic fields," Bull. Am. Phys. Soc. 46, 283 (2001).

${ }^{2}$ J. C. Maxwell, A Treatise on Electricity and Magnetism (Clarendon, Oxford, 1873), Art. 490.

${ }^{3}$ C. F. Varley, "Some experiments on the discharge of electricity through rarefied media and the atmosphere," Proc. R. Soc. London 19, 236-242 (1871).

${ }^{4}$ A. Schuster, "Experiments on the discharge of electricity through gases. Sketch of a theory," Proc. R. Soc. London 37, 317-339 (1884).

${ }^{5}$ J. J. Thomson, "On the electric and magnetic effects produced by the motion of electrified bodies," Philos. Mag. 11, 229-249 (1881).

${ }^{6} \mathrm{O}$. Heaviside, "On the electromagnetic effects due to the motion of electrification through a dielectric," Electrical Papers (Chelsea, New York, 1970), 2nd ed., Vol. 2, pp. 504-518.

${ }^{7}$ A. Schuster, "The discharge of electricity through gases," Proc. R. Soc. London 47, 526-559 (1890).

${ }^{8}$ H. A. Lorentz, Versuch einer Theorie der electrischen und optischen Erscheinungen in bewegten Körpern (E. J. Brill, Leiden, 1895), Art. 12.

${ }^{9}$ B. Rossi, "Magnetic experiments on the cosmic rays," Nature (London) 128, 300-301 (1931).

${ }^{10}$ L. M. Mott-Smith, "On an attempt to deflect magnetically the cosmic-ray corpuscles," Phys. Rev. 39, 403-414 (1932).

${ }^{11}$ L. Alvarez, "On the interior magnetic field in iron," Phys. Rev. 45, 225226 (1934).

${ }^{12}$ W. F. G. Swann and W. E. Danforth, Jr., "Deflection of cosmic-ray secondaries in magnetized iron," Phys. Rev. 45, 565 (1934).

${ }^{13}$ F. Rasetti, "Deflection of mesons in magnetized iron," Phys. Rev. 66, 1-5 (1944).

${ }^{14}$ H. W. Fuller and M. E. Hale, "Determination of magnetization distribution in thin films using electron microscopy," J. Appl. Phys. 31, 238-248 (1960).

${ }^{15}$ H. Boersch, W. Raith, and H. Weber, "Die magnetische Ablenkung von Elektronenstrahlen in dünnen Eisenschichten," Z. Phys. 161, 1-12 (1961).

${ }^{16} \mathrm{~S}$. Yamaguchi, "Wavelength perturbation in electron diffraction for magnetic analysis," Rev. Sci. Instrum. 33, 690-691 (1962).

${ }^{17}$ M. S. Cohen, "Lorentz microscopy of small ferromagnetic particles," J. Appl. Phys. 36, 1602-1611 (1965).

${ }^{18} \mathrm{R}$. W. Whitworth and H. V. Stopes-Roe, "Experimental demonstration that the couple on a bar magnet depends on $H$, not $B$," Nature (London) 234, 31-33 (1971).

${ }^{19}$ R. F. Harrington, Introduction to Electromagnetic Engineering (McGrawHill, New York, 1958), Table C-3.

${ }^{20}$ F. N. H. Robinson, Macroscopic Electromagnetism (Pergamon, Oxford, 1973), pp. 95-96.

${ }^{21}$ F. J. Lowes, "Force on a wire in a magnetic field," Nature (London) 246, 208-209 (1973).

${ }^{22}$ W. F. Brown, Jr., N. H. Frank, E. C. Kemble, W. H. Michener, C. C. Murdock, and D. L. Webster, "The teaching of electricity and magnetism 
at the college level. I. Logical standards and critical issues," Am. J. Phys. 18, 1-25 (1950).

${ }^{23}$ W. F. Brown, Jr., N. H. Frank, E. C. Kemble, W. H. Michener, C. C. Murdock, and D. L. Webster, "The teaching of electricity and magnetism at the college level. II. Two outlines for teachers," Am. J. Phys. 18, 69-88 (1950).

${ }^{24}$ W. F. Brown, Jr., "Electric and magnetic forces: A direct calculation. I," Am. J. Phys. 19, 290-304 (1951).

${ }^{25}$ W. F. Brown, Jr., "Electric and magnetic forces: A direct calculation. II," Am. J. Phys. 19, 333-350 (1951).
${ }^{26}$ W. F. Brown, Jr., N. H. Frank, E. C. Kemble, W. H. Michener, C. C. Murdock, and D. L. Webster, "The teaching of electricity and magnetism at the college level. I. Logical standards and critical issues," Am. J. Phys. 18, 1-25 (1950), note 28.

${ }^{27}$ L. W. Casperson, "Dirac's equation in semiclassical physics," Phys. Rev. A 51, 1673-1676 (1995).

${ }^{28}$ P. Weiss, "New probe reveals unfamiliar inner proton," Sci. News (Washington, D.C.) 159, 277 (2001). 